Pacific

Journal of

Mathematics

FOLIATIONS OF A SMOOTH METRIC MEASURE SPACE BY HYPERSURFACES WITH CONSTANT $f$-MEAN CURVATURE

JUNCHEOL PYO 


\title{
FOLIATIONS OF A SMOOTH METRIC MEASURE SPACE BY HYPERSURFACES WITH CONSTANT $f$-MEAN CURVATURE
}

\author{
JUNCHEOL PYO
}

\begin{abstract}
We study smooth codimension-one foliations $\mathscr{F}$ of a smooth metric measure space whose leaves have the same constant $f$-mean curvature. Firstly, we show that all the leaves of $\mathscr{F}$ are $f$-minimal hypersurfaces when either the smooth metric measure space is compact and has nonnegative BakryÉmery Ricci curvature, or the limit of the ratio of the weighted volume of a geodesic ball $B$ and the weighted area of a geodesic sphere $\partial B$ vanishes. Secondly, we prove that every leaf of $\mathscr{F}$ is strongly $f$-stable. Lastly, we show that there is no complete proper foliation of the Gaussian space whose leaves have the same constant $f$-mean curvature. In particular, there are no foliations of $\mathbb{R}^{n+1}$ whose leaves are complete proper self-similar solutions for mean curvature flow.
\end{abstract}

\section{Introduction and the statement of results}

The study of smooth codimension-one foliations of manifolds has a long history in mathematics (see [Lawson 1974] and reference therein). In [Barbosa et al. 1987; 1991; Meeks 1988; Oshikiri 1981], there are very interesting results on foliations whose leaves have constant mean curvature. In this paper, we consider foliations of a smooth metric measure space whose leaves are hypersurfaces having the same $f$-mean curvatures. The main questions we consider here concern the rigidity and $f$-minimality of such foliations of a smooth metric measure space. Extending the classical results (i.e., when $f$ is constant) to a smooth metric measure space requires $f$ or $|\nabla f|$ to be bounded in many cases; see [Morgan 2005; Wei and Wylie 2009], for example. Our proof follows the one from the case where $f$ is constant [Barbosa et al. 1987; 1991] but without any further assumption on $f$. Moreover, for particular weight functions $f$, we get rigidity results for self-similar surfaces or translating solitons which are models for singularities of mean curvature flow.

This work was supported by a Two-Year Research Grant from Pusan National University. MSC2010: primary 53C12; secondary 53C42.

Keywords: foliation, constant $f$-mean curvature, $f$-stable, smooth metric measure space. 
Recall that a smooth metric measure space $\left(M^{n+1}, \bar{g}, f\right)$ is a smooth Riemannian manifold $\left(M^{n+1}, \bar{g}\right)$ with a positive density $e^{-f}$ used to weight the volume of domains and the area of hypersurfaces. Let $\Sigma$ be an isometrically immersed hypersurface in $\left(M^{n+1}, \bar{g}\right)$. Denote by $d v$ and $d A$ the Riemannian volume forms on $M$ and $\Sigma$ with respect to $\bar{g}$ and the induced metric $g=i^{*} \bar{g}$, respectively. Then the weighted volume and area are given by $d v_{m}=e^{-f} d v$ and $d A_{m}=e^{-f} d A$, respectively.

Smooth metric measure spaces naturally arise in various fields. The Gaussian space, i.e., Euclidean space with the Gaussian density $e^{-\pi|x|^{2}}$, appears in the study of probability and statistics. Many interesting solitons in geometric flows (e.g., self-similar solutions and translating solitons to the mean curvature flow, and Ricci solitons to the Ricci flow) are represented by $f$-minimal hypersurfaces in a smooth metric measure space (see [Bakry and Émery 1985; Cheng et al. 2012; Colding and Minicozzi 2012; Huisken and Sinestrari 1999; Morgan 2005; Pyo 2014] and the references therein).

With the upper bar, we denote the geometric quantities on the ambient space $\left(M^{n+1}, \bar{g}\right)$. For example, $\bar{\nabla}, \bar{d}, \bar{\nabla}^{2}, \bar{\triangle}, \overline{\mathrm{div}}$ and Ric, denote the Levi-Civita connection, exterior differentiation, Hessian, Laplacian, divergence and Ricci tensor of $\left(M^{n+1}, \bar{g}\right)$, respectively. For a smooth metric measure space, we naturally consider the Bakry-Émery Ricci tensor $\overline{\operatorname{Ric}}_{f}$, which is defined by

$$
\overline{\operatorname{Ric}}_{f}=\overline{\operatorname{Ric}}+\bar{\nabla}^{2} f
$$

and the $f$-Laplacian $\bar{\triangle}_{f}=\bar{\triangle}-\bar{g}(\bar{\nabla} f, \bar{\nabla})$ on $M$, which is a selfadjoint operator with respect to the weighted measure $d v_{m}$. For a smooth vector field $\xi$, the $f$-divergence of $\xi$ is defined by

$$
\overline{\operatorname{div}}_{f} \xi=e^{f} \overline{\operatorname{div}}\left(e^{-f} \xi\right) .
$$

Let $v$ be a unit normal vector field to $\Sigma$ in $M$. With the induced metric $g=i^{*} \bar{g}$ on $\Sigma$, the second fundamental form of $(\Sigma, g)$ is given by $A(X, Y)=g\left(\bar{\nabla}_{X} Y, v\right)$ for any two tangent vectors $X$ and $Y$ on $\Sigma$, and the mean curvature by $H=\operatorname{tr}(A)$. For the hypersurface $\Sigma$ in $(M, \bar{g}, f)$, we define the $f$-mean curvature $H_{f}$ with respect to $v$ as follows:

$$
H_{f}=H+\bar{g}(\bar{\nabla} f, v)
$$

which is obtained by the first variation formula of the weighted area. For $(\Sigma, g), \nabla$, $d, \triangle$ and div denote the Levi-Civita connection, exterior differentiation, Laplacian and divergence on $\Sigma$, respectively.

The following is proved for foliations of a compact smooth metric measure space with nonnegative Bakry-Émery Ricci curvature: 
Theorem 2. Let $\left(M^{n+1}, \bar{g}, f\right)$ be a compact smooth metric measure space with nonnegative Bakry-Émery Ricci curvature and F a codimension-one smooth foliation of $M$ whose leaves have the same constant $f$-mean curvature. Then every leaf of $\mathscr{F}_{\mathrm{F}}$ is a totally geodesic and $f$-minimal hypersurface with vanishing Bakry-Émery Ricci curvature in the normal direction.

In a smooth metric measure space $\left(M^{n+1}, \bar{g}, f\right)$, we define the ratio

$$
\Lambda_{f}(R, p)=\frac{\operatorname{vol}_{f}\left(\partial B_{p}(R)\right)}{\overline{\operatorname{vol}_{f}\left(B_{p}(R)\right)}},
$$

where $\overline{\operatorname{vol}}_{f}\left(B_{p}(R)\right)$ and $\operatorname{vol}_{f}\left(\partial B_{p}(R)\right)$ are the weighted volume of the geodesic ball $B_{p}(R)$ and the geodesic sphere $\partial B_{p}(R)$ for a point $p$, respectively. For smooth metric measure spaces of vanishing $\Lambda_{f}(R, p)$ as $R \rightarrow \infty$, we show:

Theorem 6. Let $\mathscr{F}$ be an orientable codimension-one foliation of $\left(M^{n+1}, \bar{g}, f\right)$ such that every orientable leaf $L$ of $\mathscr{F}$ has the same constant $f$-mean curvature. If $\lim _{R \rightarrow \infty}=\Lambda_{f}(R, p)=0$ for some $p \in M$, then leaves of $\mathscr{F}$ are $f$-minimal hypersurfaces of $\left(M^{n+1}, \bar{g}, f\right)$.

We remark that the Gaussian space and $\left(\mathbb{R}^{n+1}, d s_{0}, f=x_{n+1}\right)$ enjoy the property that, for any point $p$, the ratio $\Lambda_{f}(R, p)$ vanishes as $R \rightarrow \infty$.

In Section 3, we prove:

Theorem 11. Let $\left(M^{n+1}, \bar{g}, f\right)$ be an orientable smooth metric measure space and $\mathscr{F}$ a smooth codimension-one foliation of $M$ by orientable leaves. If each leaf of $\mathscr{F}_{\text {has }}$ the same constant $f$-mean curvature, then each leaf of $\mathscr{F}^{\text {is strongly }}$ $f$-stable.

Theorem 13. There are no complete proper foliations in the Gaussian space $\left(\mathbb{R}^{n+1}, d s_{0}, f=|X|^{2} / 2\right)$ whose leaves have the same constant $f$-mean curvature. In particular, there are no foliations of $\mathbb{R}^{n+1}$ whose leaves are complete proper self-similar solutions for mean curvature flow.

\section{Foliation whose leaves are $f$-minimal hypersurfaces}

Let us start with the key lemma about the $f$-divergence of $\bar{\nabla}_{v} v$. The proof is analogous to that of Proposition 2.14 in [Barbosa et al. 1991], but we include its proof in the Appendix for the sake of completeness.

Lemma 1. Let F्F be a smooth codimension-one foliation of a smooth metric measure space $\left(M^{n+1}, \bar{g}, f\right)$ and $v$ a unit normal vector field to the leaves of $\mathscr{F}$ in some open subset $U$ of $M$. Define a tangent vector field $\xi=\bar{\nabla}_{v} v$. Then on $U$, we have:

(a) $\overline{\operatorname{div}}_{f} v=-H_{f}$;

(b) $\overline{\operatorname{div}}_{f} \xi=\operatorname{div}_{f} \xi-|\xi|_{g}^{2}$; 
(c) $\operatorname{div}_{f} \xi=|\xi|_{g}^{2}+|A|^{2}+\overline{\operatorname{Ric}}_{f}(v, v)-v H_{f}$.

Theorem 2. Let $\left(M^{n+1}, \bar{g}, f\right)$ be a compact smooth metric measure space with nonnegative Bakry-Émery Ricci curvature and F a codimension-one smooth foliation of $M$ whose leaves have the same constant $f$-mean curvature. Then every leaf of $\mathscr{F}$ is a totally geodesic and $f$-minimal hypersurface with vanishing Bakry-Émery Ricci curvature in the normal direction.

Proof. Since $H_{f}$ is constant in $M, v\left(H_{f}\right) \equiv 0$. Then Lemma 1(c) implies that

$$
\operatorname{div}_{f} \xi=|A|^{2}+|\xi|_{g}^{2}+\overline{\operatorname{Ric}}_{f}(v, v)
$$

on any leaf of $\mathscr{F}$, and therefore Lemma 1(b) implies that

$$
\overline{\operatorname{div}}_{f} \xi=|A|^{2}+\overline{\operatorname{Ric}}_{f}(v, v) .
$$

Recall that $d v_{m}=e^{-f} d v$. Integrating both sides and applying Stokes' theorem on $M$, we get

$$
0=\int_{M} \overline{\operatorname{div}}_{f} \xi d v_{m}=\int_{M}|A|^{2}+\overline{\operatorname{Ric}}_{f}(v, v) d v_{m},
$$

that is, $|A|^{2}=0$ and $\overline{\operatorname{Ric}}_{f}(v, v)=0$ on $M$. Therefore, every leaf is a totally geodesic hypersurface with vanishing Bakry-Émery Ricci curvature in the normal direction.

Since $M$ is compact, there exists a point $m \in M$ such that $f(m)=\max _{M} f$. At $m$, we have $\bar{\nabla} f(m)=0$. Therefore $H_{f}(L)=-\bar{g}(\bar{\nabla} f(m), v)=0$, where $L$ is the leaf which contains the point $m$. So, $H_{f} \equiv 0$ on any leaf of $\mathscr{F}$. This completes the proof.

Remark 3. (1) The compactness condition in Theorem 2 is necessary. The smooth metric measure space $\left(\mathbb{R}^{n+1}, d s_{0}, f=x_{n+1}\right)$ has vanishing Bakry-Émery Ricci curvature and is noncompact. Translating solitons under the mean curvature flow do not change shape and are just translated in a direction with a constant speed. Up to rotating and scaling, they are represented by $x_{n+1}$-minimal hypersurfaces in the smooth metric measure space $\left(\mathbb{R}^{n+1}, d s_{0}, f=x_{n+1}\right)$ (see [Huisken and Sinestrari 1999]). By [Altschuler and Wu 1994] for $n=2$, and [Gui, Jian and Ju 2010] for $n \geq 3$, there exists an entire rotationally symmetric strictly convex graphical hypersurface $U$, which gives a foliation by $x_{n+1}$-minimal hypersurfaces. But clearly $U$ is not a totally geodesic hypersurface.

(2) The theorem of (Bonnet and) Myers [1941] says that a complete Riemannian manifold $M$ is compact when $M$ has Ricci curvature bounded from below by a positive constant. But this does not hold in general for a smooth metric measure space. One such example is the Gaussian space $\left(\mathbb{R}^{n+1}, d s_{0}, f=|X|^{2} / 2\right)$. There are some generalizations of the Bonnet-Myers theorem with conditions on $f$ [Morgan 2005; Wei and Wylie 2009]. 
Theorem 4. Let $\left(M^{n+1}, \bar{g}, f\right)$ be a smooth metric measure space with positive Bakry-Émery Ricci curvature. Any smooth codimension-one foliation of $M$ whose leaves have the same constant $f$-mean curvature cannot have a compact leaf.

Proof. Suppose that, on the contrary, there exists a compact leaf $L$ in the foliation $\mathscr{F}$. Lemma 1(c) implies that

$$
\operatorname{div}_{f} \xi=|\xi|_{g}^{2}+|A|^{2}+\overline{\operatorname{Ric}}_{f}(v, v)
$$

on $L$. Weighting both sides by $d A_{m}=e^{-f} d A$, integrating, and applying Stokes' theorem on $L$, we get a contradiction.

Let $\mathscr{F}$ be a smooth orientable codimension-one foliation and $L$ a leaf of $\mathscr{F}$. The weighted volume element $d A_{m}=\varphi_{f}$ of $L$ is defined as follows:

$$
\varphi_{f}\left(X_{1}, \ldots, X_{n}\right)=e^{-f} g\left(X_{1} \wedge \cdots \wedge X_{n}, v\right),
$$

where the $X_{i}$ are tangent vector fields $(i=1, \ldots, n)$.

With a positively oriented frame field $\left\{e_{1}, \ldots, e_{n}, e_{n+1}=v\right\}$, and its dual coframe $\left\{\omega_{1}, \ldots, \omega_{n+1}\right\}$, the weighted volume elements $d A_{m}=\varphi_{f}$ and $d v_{m}=\Phi_{m}$ are expressed by

$$
\begin{aligned}
\varphi_{f} & =e^{-f} \omega_{1} \wedge \cdots \wedge \omega_{n}, \\
\Phi_{f} & =e^{-f} \omega_{1} \wedge \cdots \wedge \omega_{n+1} .
\end{aligned}
$$

Both these weighted volume elements are related by the Rummler-type identity [Rummler 1979] as follows:

Lemma 5. Let $\left(M^{n+1}, \bar{g}, f\right)$ be an orientable smooth metric measure space and $\mathscr{F}$ a smooth codimension-one foliation of $M$ by orientable leaves. Then

$$
\bar{d} \varphi_{f}=(-1)^{n+1} H_{f} \Phi_{f},
$$

where $\varphi_{f}$ is a weighted volume element of leaves of $\mathscr{F}$.

Proof. Taking exterior differentiation on $\varphi_{f}$, we have

$$
\bar{d} \varphi_{f}=-e^{-f} \bar{d} f \wedge \omega_{1} \wedge \cdots \wedge \omega_{n}+e^{-f} \bar{d}\left(\omega_{1} \wedge \cdots \wedge \omega_{n}\right) .
$$

Since

$$
\bar{d} f=e_{1} f \omega_{1}+\cdots+e_{n+1} f \omega_{n+1}
$$

and

$$
\bar{d}\left(\omega_{1} \wedge \cdots \wedge \omega_{n}\right)=(-1)^{n+1} H \omega_{1} \wedge \cdots \wedge \omega_{n+1},
$$

we have

$$
\begin{aligned}
\bar{d} \varphi_{f} & =(-1)^{n+1} e^{-f}\left(e_{n+1} f\right) \omega_{1} \wedge \cdots \wedge \omega_{n+1}+(-1)^{n+1} e^{-f} H \omega_{1} \wedge \cdots \wedge \omega_{n+1} \\
& =(-1)^{n+1} H_{f} \Phi_{f} .
\end{aligned}
$$


Let $p$ be a point in $M$, and $B_{p}(R)$ a geodesic ball in $(M, \bar{g})$ of radius $R$ centered at $p$. The boundary of $B_{p}(R)$ is denoted by $\partial B_{p}(R)$. Define the ratio of the weighted volume of $B_{p}(R)$ and $\partial B_{p}(R)$ as follows:

$$
\Lambda_{f}(R, p)=\frac{\operatorname{vol}_{f}\left(\partial B_{p}(R)\right)}{\overline{\operatorname{vol}}_{f}\left(B_{p}(R)\right)},
$$

where $\overline{\operatorname{vol}}_{f}\left(B_{p}(R)\right)$ and $\operatorname{vol}_{f}\left(\partial B_{p}(R)\right)$ are the weighted volumes of $B_{p}(R)$ and $\partial B_{p}(R)$, respectively.

Theorem 6. Let $\mathscr{F}$ be an orientable codimension-one foliation of $\left(M^{n+1}, \bar{g}, f\right)$ such that every orientable leaf $L$ of $\mathscr{F}$ has the same constant $f$-mean curvature. If $\lim _{R \rightarrow \infty} \Lambda_{f}(R, p)=0$ for some $p \in M$, then leaves of F are $f$-minimal hypersurfaces of $\left(M^{n+1}, \bar{g}, f\right)$.

Proof. Suppose not. Then, choosing a normal vector field, we may assume that

$$
(-1)^{n+1} H_{f}>0 \text {. }
$$

Let $\sigma_{f}$ be a weighted volume element of $\partial B_{p}(R)$. That is, for a local orthonormal frame field $\left\{X_{1}, \ldots, X_{n}\right\}$ which is tangent to $\partial B_{p}(R)$,

$$
\sigma_{f}\left(X_{1}, \ldots, X_{n}\right)=e^{-f} \text {. }
$$

On $\partial B_{p}(R)$, we have $\varphi_{f} \leq \sigma_{f}$.

By Lemma 5, we have

$$
\begin{aligned}
\overline{\operatorname{vol}}_{f}\left(B_{p}(R)\right) & =\int_{B_{p}(R)} \Phi_{f}=\int_{B_{p}(R)} \frac{(-1)^{n+1}}{H_{f}} \bar{d} \varphi_{f} \\
& =\frac{(-1)^{n+1}}{H_{f}} \int_{\partial B_{p}(R)} \varphi_{f} \\
& \leq \frac{(-1)^{n+1}}{H_{f}} \int_{\partial B_{p}(R)} \sigma_{f} \\
& =\frac{(-1)^{n+1}}{H_{f}} \operatorname{vol}_{f}\left(\partial B_{p}(R)\right) .
\end{aligned}
$$

Therefore

$$
0<(-1)^{n+1} H_{f} \leq \frac{\operatorname{vol}_{f}\left(\partial B_{p}(R)\right)}{\overline{\operatorname{vol}}_{f}\left(B_{p}(R)\right)}=\Lambda_{f}(R, p) .
$$

As $R$ goes to $\infty$, we get a contradiction, and this completes the proof.

Let $X=\left(x_{1}, \ldots, x_{n+1}\right)$ be the position vector in $\mathbb{R}^{n+1}$ and $|X|^{2}=x_{1}^{2}+\cdots+x_{n+1}^{2}$. Self-shrinkers under the mean curvature flow in $\mathbb{R}^{n+1}$ are represented by $|X|^{2} / 2$ minimal hypersurfaces in the Gaussian space $\left(\mathbb{R}^{n+1}, d s_{0}, f=|X|^{2} / 2\right.$ ) (see [Colding and Minicozzi 2012]). 
By direct computation,

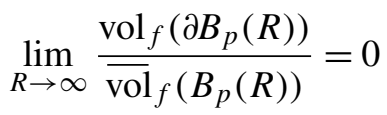

in the Gaussian space, and therefore the following corollary is obtained:

Corollary 7. Let $\mathscr{F}$ be an orientable codimension-one foliation of the Gaussian space such that every orientable leaf $L$ of $F_{F}$ has the same constant $f$-mean curvature. Then leaves of $\mathscr{F}$ are self-shrinkers.

By direct computation,

$$
\lim _{R \rightarrow \infty} \frac{\operatorname{vol}_{f}\left(\partial B_{p}(R)\right)}{\overline{\operatorname{vol}}_{f}\left(B_{p}(R)\right)}=0
$$

also holds in $\left(\mathbb{R}^{n+1}, d s_{0}, f=x_{n+1}\right)$, and therefore the following corollary is obtained:

Corollary 8. Let $\mathscr{F}$ be an orientable codimension-one foliation of $\left(\mathbb{R}^{n+1}, d s_{0}, f=\right.$ $\left.x_{n+1}\right)$ such that every orientable leaf $L$ of $\mathscr{F}$ has the same constant $f$-mean curvature. Then leaves of $\mathscr{F}$ are translating solitons.

Let $\Sigma$ be a hypersurface in $\left(\mathbb{R}^{n+1}, d s_{0}, f=x_{n+1}\right)$ and $H_{f}$ its $f$-mean curvature. Translating $\Sigma$ in the direction of $e_{n+1}=(0, \ldots, 0,1) \in \mathbb{R}^{n+1}$, the $f$-mean curvature does not change. Using this property we get a Bernstein-type theorem for constant $f$-mean curvature surfaces.

Corollary 9. Let $x_{n+1}=F\left(x_{1}, \ldots, x_{n}\right)$ be a hypersurface of constant $f$-mean curvature defined on $\left\{x_{n+1}=0\right\}$ in $\left(\mathbb{R}^{n+1}, d s_{0}, f=x_{n+1}\right)$. Then the $f$-mean curvature must be zero.

Proof. Consider the graph graph $(F)$ of the function $F$. The family

$$
\left\{\operatorname{graph}(F)+t e_{n+1}\right\}_{t \in \mathbb{R}}
$$

gives a foliation whose leaves are hypersurfaces in $\left(\mathbb{R}^{n+1}, d s_{0}, f=x_{n+1}\right)$ with the same constant $f$-mean curvature. From Corollary $8, H_{f}$ vanishes.

When $f$ is a constant, then Corollary 9 becomes the corollary from p. 82 of [Chern 1965].

\section{Stability of foliations whose leaves have the same constant $f$-mean curvature}

Let $\Sigma^{n}$ be a constant $f$-mean curvature hypersurface in $\left(M^{n+1}, \bar{g}, f\right)$. The $f$ stability operator $L_{f}$ is defined as

$$
L_{f}:=\triangle_{f}+|A|^{2}+\overline{\operatorname{Ric}}_{f}(v, v),
$$


where $v$ is a unit normal vector field of $\Sigma$ (see [Cheng et al. 2012; Colding and Minicozzi 2012; Espinar 2012]).

Definition 10. A two-sided hypersurface $\Sigma$ in $\left(M^{n+1}, \bar{g}, f\right)$ with constant $f$-mean curvature is said to be strongly $f$-stable if for any compactly supported smooth function $u \in C_{c}^{\infty}(\Sigma)$, it satisfies

$$
-\int_{\Sigma} u L_{f} u d A_{m}=\int_{\Sigma}|\nabla u|^{2}-\left(|A|^{2}+\overline{\operatorname{Ric}}_{f}(v, v)\right) u^{2} d v_{m} \geq 0 .
$$

If $\Sigma$ is an $f$-minimal hypersurface, then strong $f$-stability is equivalent to usual $f$-stability.

Theorem 11. Let $\left(M^{n+1}, \bar{g}, f\right)$ be an orientable smooth metric measure space and $\mathscr{F}$ a smooth codimension-one foliation of $M$ by orientable leaves. If each leaf of $\mathscr{F}$ has the same constant $f$-mean curvature, then each leaf of $\mathscr{F}$ is strongly $f$-stable.

Proof. Let $L$ be a leaf of $\mathscr{F}$ and $u$ a smooth real-valued function which is compactly supported on a domain $D$ in $L$ (therefore, $u$ is zero on $\partial D$ ). Then

$$
-u L_{f} u=-u \triangle_{f} u-\left(|A|^{2}+\overline{\operatorname{Ric}}_{f}(v, v)\right) u^{2}=-u \triangle_{f} u+u^{2} \operatorname{div}_{f} \xi+u^{2}|\xi|_{g}^{2} .
$$

Here we apply equation (c) in Lemma 1.

Since $\operatorname{div}_{f}\left(u^{2} \xi\right)=2 u g(\nabla u, \xi)+u^{2} \operatorname{div}_{f} \xi$, we get

$$
-u L_{f} u=-u \triangle_{f} u-2 u g(\nabla u, \xi)+u^{2}|\xi|_{g}^{2}+\operatorname{div}_{f}\left(u^{2} \xi\right) .
$$

Weighting both sides by $d v_{m}$, integrating over $D$, and applying Stokes' theorem twice for the first and the last terms, we have

$$
\begin{aligned}
\int_{D}-u L_{f} u d v_{m} & =\int_{D}-u \triangle_{f} u-2 u g(\nabla u, \xi)+u^{2}|\xi|_{g}^{2}+\operatorname{div}_{f}\left(u^{2} \xi\right) d v_{m} \\
& =\int_{D}|\nabla u|_{g}^{2}-2 u g(\nabla u, \xi)+u^{2}|\xi|_{g}^{2} d v_{m} \\
& =\int_{D}|\nabla u-u \xi|_{g}^{2} d v_{m} \geq 0 .
\end{aligned}
$$

Since $u$ is an arbitrary function, we conclude that $L$ is $f$-stable.

Remark 12. Let $\Sigma$ be a graph over a domain $\Omega \subset\left\{x_{n+1}=0\right\}$ in $\left(\mathbb{R}^{n+1}, d s_{0}, f=\right.$ $\left.x_{n+1}\right)$ having constant $f$-mean curvature. Denote $\Sigma_{t}=\Sigma+t e_{n+1}, t \in \mathbb{R}$. Then, by Theorem 11, every $\Sigma_{t}$ is strongly $f$-stable. For example, the family of "grim reapers" $\Sigma_{t}=\left\{\left(x_{1}, \ldots, x_{n}, t-\ln \cos x_{1}:\left|x_{1}\right|<\pi / 2\right)\right\}$ is a foliation in the open manifold $\left.\left\{\left(x_{1}, \ldots, x_{n+1}\right):\left|x_{1}\right|<\pi / 2\right)\right\}$ in $\left(\mathbb{R}^{n+1}, d s_{0}, f=x_{n+1}\right)$. So, every grim reaper is strongly $f$-stable. 
Let $\mathscr{F}$ be a foliation of the Gaussian space $\left(\mathbb{R}^{n+1}, d s_{0}, f=|X|^{2} / 2\right)$. If every leaf of $\mathscr{F}$ is proper (respectively, complete), then $\mathscr{F}$ is said to be proper (respectively, complete).

Theorem 13. There are no complete proper foliations in the Gaussian space $\left(\mathbb{R}^{n+1}, d s_{0}, f=|X|^{2} / 2\right)$ whose leaves have the same constant $f$-mean curvature. In particular, there are no foliations of $\mathbb{R}^{n+1}$ whose leaves are complete proper self-similar solutions for mean curvature flow.

Recall Colding and Minicozzi's result for self-shrinkers in the Gaussian space:

Theorem 14 [Colding and Minicozzi 2012]. There are no f-stable complete selfshrinkers without boundary and with polynomial volume growth in the Gaussian space.

Proof of Theorem 13. Suppose, on the contrary, that $\mathscr{F}$ is a complete, proper foliation whose leaves have the same $f$-mean curvature. By Corollary 7 and foliated structure, every leaf $L$ of $\mathscr{F}$ is a self-shrinker without boundary. By Theorem 11, $L$ is $f$-stable. Cheng and Zhou [2013] proved that for self-shrinkers, properness is equivalent to polynomial volume growth. Therefore, $L$ is an $f$-stable complete self-shrinker without boundary and with polynomial volume growth in the Gaussian space. This contradicts Theorem 14.

\section{Appendix}

Let $\mathscr{F}$ be a smooth codimension-one foliation of a smooth metric measure space $\left(M^{n+1}, \bar{g}, f\right)$. On a leaf of $\mathscr{F}$, the induced metric is denoted by $g=i^{*} \bar{g}$, where $i$ is the inclusion map. Let $\left\{e_{1}, \ldots, e_{n+1}\right\}$ be a locally defined orthonormal frame field of the tangent bundle of $M$ such that $e_{n+1}$ is normal to the leaves of $\mathscr{F}$. Let us denote the dual coframe field by $\left\{\omega_{1}, \ldots, \omega_{n+1}\right\}$, that is, $\omega_{A}\left(e_{B}\right)=\delta_{A B}$.

The connection one-forms $\omega_{A B}$ are given by exterior differentiation $\bar{d}$ of the $\omega_{A}$, and are uniquely defined by Cartan's first structure equations:

$$
\bar{d} \omega_{A}=\omega_{A B} \wedge \omega_{B}, \quad \omega_{A B}+\omega_{B A}=0 .
$$

Cartan's second structure equations yield the curvature tensor

$$
\bar{d} \omega_{A B}=\omega_{A C} \wedge \omega_{C B}+\Omega_{A B},
$$

where

$$
\Omega_{A B}=\frac{1}{2} R_{A B C D} \omega_{D} \wedge \omega_{C} .
$$

Throughout, we adopt Einstein's convention and the following indexing convention:

$$
1 \leq i, j, k, l \leq n, \quad 1 \leq A, B, C, D \leq n+1 .
$$


The second fundamental form $A$ of the leaves of $\mathscr{F}$ is given by

$$
\omega_{n+1 i}=-h_{i j} \omega_{j}
$$

where

$$
h_{i j}=\bar{g}\left(A\left(e_{i}, e_{j}\right), e_{n+1}\right)=\bar{g}\left(\bar{\nabla}_{e_{i}} e_{j}, e_{n+1}\right) .
$$

The mean curvature is $H=\sum_{i} h_{i i}$.

Proof of Lemma 1. Consider an adapted orthonormal frame field $\left\{e_{1}, \ldots, e_{n+1}\right\}$ on $U$ such that $e_{n+1}=v$. We have

$$
\begin{aligned}
\overline{\operatorname{div}}_{f} v & =e^{f} \overline{\operatorname{div}}\left(e^{-f} v\right)=e^{f} \bar{g}\left(\bar{\nabla}_{e_{A}} e^{-f} v, e_{A}\right) \\
& =-\bar{g}\left(e_{A} f v, e_{A}\right)+\bar{g}\left(\bar{\nabla}_{e_{A}} v, e_{A}\right) \\
& =-\bar{g}(\bar{\nabla} f, v)-H=-H_{f} .
\end{aligned}
$$

Therefore, the equation (a) holds.

Furthermore,

$$
\begin{aligned}
\overline{\operatorname{div}}_{f} \xi & =e^{f} \overline{\operatorname{div}}\left(e^{-f} \xi\right) \\
& =e^{f} g\left(\bar{\nabla}_{e_{i}} e^{-f} \xi, e_{i}\right)+\bar{g}\left(\bar{\nabla}_{e_{n+1}} e^{-f} \xi, e_{n+1}\right) \\
& =\operatorname{div}_{f} \xi-\bar{g}\left(\xi, \bar{\nabla}_{e_{n+1}} e_{n+1}\right)=\operatorname{div}_{f} \xi-|\xi|_{g}^{2}
\end{aligned}
$$

Therefore, the equation (b) holds.

Since $\bar{d} u=d u+e_{n+1}(u) \omega_{n+1}$ for any smooth function $u$ in $U$, from (2) we get

$$
\omega_{n+1 i}=-h_{i j} \omega_{j}+g\left(\xi, e_{i}\right) \omega_{n+1} .
$$

On the one hand, from (1), we have

$$
\begin{aligned}
\bar{d} \omega_{n+1 i}= & \omega_{n+1 j} \wedge \omega_{j i}+R_{n+1 i n+1 k} \omega_{k} \wedge \omega_{n+1}+\frac{1}{2} R_{n+1 i j k} \omega_{k} \wedge \omega_{j} \\
= & \left(-h_{j k} \omega_{j i}\left(e_{n+1}\right)-g\left(\xi, e_{i}\right) \omega_{j i}\left(e_{k}\right)+R_{n+1 i n+1 k}\right) \omega_{k} \wedge \omega_{n+1} \\
& + \text { terms with } \omega_{k} \wedge \omega_{l} .
\end{aligned}
$$

On the other hand, from (3),

$$
\begin{gathered}
\bar{d} \omega_{n+1 i}=-\left(d h_{i j}+e_{n+1} h_{i j} \omega_{n+1}\right) \wedge \omega_{j}-h_{i j} \omega_{j k} \wedge \omega_{k}-h_{i j} \omega_{j n+1} \wedge \omega_{n+1} \\
+\bar{d} g\left(\xi, e_{i}\right) \omega_{n+1}+g\left(\xi, e_{1}\right) \omega_{n+1 j} \wedge \omega_{j} \\
=\left(e_{n+1} h_{i k}+h_{i j} \omega_{j k}\left(e_{n+1}\right)-h_{i j} h_{j k}+d g\left(\xi, e_{i}\right)\left(e_{k}\right)\right. \\
\left.-g\left(\xi, e_{i}\right) g\left(\xi, e_{j}\right)\right) \omega_{k} \wedge \omega_{n+1}+\text { terms with } \omega_{k} \wedge \omega_{l}
\end{gathered}
$$

By investigating both of the coefficients of $\omega_{k} \wedge \omega_{n+1}$ in $\bar{d} \omega_{n+1 i}$, we have 
(4)

$$
\begin{array}{r}
g\left(\xi, e_{i}\right) g\left(\xi, e_{k}\right)+h_{i j} h_{j k}+R_{n+1 i n+1 k}-\left(d h_{i k}+h_{i j} \omega_{j k}+h_{j k} \omega_{j i}\right)\left(e_{n+1}\right) \\
=\left(\bar{d} g\left(\xi, e_{i}\right)+g\left(\xi, e_{i}\right) \omega_{j i}\right)\left(e_{k}\right) .
\end{array}
$$

Since $\bar{d} g\left(\xi, e_{i}\right)\left(e_{k}\right)=d g\left(\xi, e_{j}\right)\left(e_{k}\right)$ and $g\left(\nabla_{e_{i}} \xi, e_{i}\right)=d g\left(\xi, e_{i}\right)+g\left(\xi, e_{j}\right) \omega_{j i}\left(e_{i}\right)$,

$$
\begin{aligned}
\operatorname{div}_{f} \xi & =e^{f} \operatorname{div}\left(e^{-f} \xi\right)=\operatorname{div} \xi-g(\nabla f, \xi) \\
& =\sum_{i}\left(d g\left(\xi, e_{i}\right)+g\left(\xi, e_{j}\right) \omega_{j i}\right)\left(e_{i}\right)-g(\nabla f, \xi) \\
& =\sum_{i} g\left(\xi, e_{i}\right)^{2}+|A|^{2}+\overline{\operatorname{Ric}}(v, v)-v H-g(\nabla f, \xi) \\
& =|\xi|_{g}^{2}+|A|^{2}+\overline{\operatorname{Ric}}(v, v)-v H-v g(\bar{\nabla} f, v)+v g(\bar{\nabla} f, v)-g(\nabla f, \xi) \\
& =|\xi|_{g}^{2}+|A|^{2}-v H_{f}+\overline{\operatorname{Ric}}(v, v)+\bar{\nabla}^{2} f(v, v) \\
& =|\xi|_{g}^{2}+|A|^{2}-v H_{f}+\overline{\operatorname{Ric}}_{f}(v, v) .
\end{aligned}
$$

This completes the proof.

\section{References}

[Altschuler and Wu 1994] S. J. Altschuler and L. F. Wu, "Translating surfaces of the non-parametric mean curvature flow with prescribed contact angle", Calc. Var. Partial Differential Equations 2:1 (1994), 101-111. MR 97b:58032 Zbl 0812.35063

[Bakry and Émery 1985] D. Bakry and M. Émery, "Diffusions hypercontractives", pp. 177-206 in Séminaire de Probabilités, XIX (Strasbourg, 1983-1984), edited by J. Azéma and M. Yor, Lecture Notes in Math. 1123, Springer, Berlin, 1985. MR 88j:60131 Zbl 0561.60080

[Barbosa et al. 1987] J. L. M. Barbosa, J. M. Gomes, and A. M. Silveira, "Foliation of 3-dimensional space forms by surfaces with constant mean curvature", Bol. Soc. Brasil. Mat. 18:2 (1987), 1-12. MR 90j:53054 Zbl 0747.53029

[Barbosa et al. 1991] J. L. M. Barbosa, K. Kenmotsu, and G. Oshikiri, "Foliations by hypersurfaces with constant mean curvature", Math. Z. 207:1 (1991), 97-107. MR 92b:53034 Zbl 0731.53033

[Cheng and Zhou 2013] X. Cheng and D. Zhou, "Volume estimate about shrinkers", Proc. Amer. Math. Soc. 141:2 (2013), 687-696. MR 2996973 Zbl 1262.53030

[Cheng et al. 2012] X. Cheng, T. Mejia, and D. Zhou, "Eigenvalue estimate and compactness for closed $f$-minimal surfaces", preprint, 2012. arXiv 1210.8448

[Chern 1965] S.-S. Chern, "On the curvatures of a piece of hypersurface in Euclidean space", $A b h$. Math. Sem. Univ. Hamburg 29 (1965), 77-91. MR 32 \#6376 Zbl 0147.20901

[Colding and Minicozzi 2012] T. H. Colding and W. P. Minicozzi, II, "Generic mean curvature flow, I: Generic singularities", Ann. of Math. (2) 175:2 (2012), 755-833. MR 2993752 Zbl 1239.53084

[Espinar 2012] J. M. Espinar, "Manifolds with density, applications and gradient Schrödinger operators", preprint, 2012. arXiv 1209.6162

[Gui, Jian and Ju 2010] C. Gui, H. Jian, and H. Ju, "Properties of translating solutions to mean curvature flow”, Discrete Contin. Dyn. Syst. 28:2 (2010), 441-453. MR 2011h:35081 Zbl 1193.35085

[Huisken and Sinestrari 1999] G. Huisken and C. Sinestrari, "Convexity estimates for mean curvature flow and singularities of mean convex surfaces", Acta Math. 183:1 (1999), 45-70. MR 2001c:53094 Zbl 0992.53051 
[Lawson 1974] H. B. Lawson, Jr., "Foliations", Bull. Amer. Math. Soc. 80 (1974), 369-418. MR 49 \#8031 Zbl 0293.57014

[Meeks 1988] W. H. Meeks, III, "The topology and geometry of embedded surfaces of constant mean curvature”, J. Differential Geom. 27:3 (1988), 539-552. MR 89h:53025 Zbl 0617.53007

[Morgan 2005] F. Morgan, "Manifolds with density", Notices Amer. Math. Soc. 52:8 (2005), 853-858. MR 2006g:53044 Zbl 1118.53022

[Myers 1941] S. B. Myers, "Riemannian manifolds with positive mean curvature", Duke Math. J. 8 (1941), 401-404. MR 3,18f Zbl 0025.22704

[Oshikiri 1981] G.-I. Oshikiri, "A remark on minimal foliations”, Tôhoku Math. J. (2) 33:1 (1981), 133-137. MR 83b:57017 Zbl 0437.57013

[Pyo 2014] J. Pyo, “Compact translating solitons with non-empty planar boundary”, preprint, 2014.

[Rummler 1979] H. Rummler, "Quelques notions simples en géométrie Riemannienne et leurs applications aux feuilletages compacts", Comment. Math. Helv. 54:2 (1979), 224-239. MR 80m:57021 Zbl 0409.57026

[Wei and Wylie 2009] G. Wei and W. Wylie, "Comparison geometry for the Bakry-Emery Ricci tensor”, J. Differential Geom. 83:2 (2009), 377-405. MR 2011a:53064 Zbl 1189.53036

Received May 16, 2013. Revised April 8, 2014.

JUNCHEOL PYO

DEPARTMENT OF MATHEMATICS

PUSAN NATIONAL UNIVERSITY

BUSAN 609-735

SOUTH KOREA

jcpyo@pusan.ac.kr

and

SCHOOL OF MATHEMATICS

Korea Institute FOR AdVANCED STUdy (KIAS)

SEOUL 130-722

SOUTH KOREA 


\title{
PACIFIC JOURNAL OF MATHEMATICS
}

\author{
msp.org/pjm
}

Founded in 1951 by E. F. Beckenbach (1906-1982) and F. Wolf (1904-1989)

\section{EDITORS}

Don Blasius (Managing Editor)

Department of Mathematics

University of California

Los Angeles, CA 90095-1555

blasius@math.ucla.edu

\author{
Paul Balmer \\ Department of Mathematics \\ University of California \\ Los Angeles, CA 90095-1555 \\ balmer@math.ucla.edu \\ Robert Finn \\ Department of Mathematics \\ Stanford University \\ Stanford, CA 94305-2125 \\ finn@math.stanford.edu \\ Sorin Popa \\ Department of Mathematics \\ University of California \\ Los Angeles, CA 90095-1555 \\ popa@math.ucla.edu
}

\author{
Vyjayanthi Chari \\ Department of Mathematics \\ University of California \\ Riverside, CA 92521-0135 \\ chari@math.ucr.edu \\ Kefeng Liu \\ Department of Mathematics \\ University of California \\ Los Angeles, CA 90095-1555 \\ liu@math.ucla.edu \\ Jie Qing \\ Department of Mathematics \\ University of California \\ Santa Cruz, CA 95064 \\ qing@ cats.ucsc.edu
}

\section{PRODUCTION}

Silvio Levy, Scientific Editor, production@msp.org

\section{SUPPORTING INSTITUTIONS}

ACADEMIA SINICA, TAIPEI

CALIFORNIA INST. OF TECHNOLOGY

INST. DE MATEMÁTICA PURA E APLICADA

KEIO UNIVERSITY

MATH. SCIENCES RESEARCH INSTITUTE

NEW MEXICO STATE UNIV.

OREGON STATE UNIV.

\author{
STANFORD UNIVERSITY \\ UNIV. OF BRITISH COLUMBIA \\ UNIV. OF CALIFORNIA, BERKELEY \\ UNIV. OF CALIFORNIA, DAVIS \\ UNIV. OF CALIFORNIA, LOS ANGELES \\ UNIV. OF CALIFORNIA, RIVERSIDE \\ UNIV. OF CALIFORNIA, SAN DIEGO \\ UNIV. OF CALIF., SANTA BARBARA
}

\author{
Daryl Cooper \\ Department of Mathematics \\ University of California \\ Santa Barbara, CA 93106-3080 \\ cooper@math.ucsb.edu \\ Jiang-Hua Lu \\ Department of Mathematics \\ The University of Hong Kong \\ Pokfulam Rd., Hong Kong \\ jhlu@maths.hku.hk \\ Paul Yang \\ Department of Mathematics \\ Princeton University \\ Princeton NJ 08544-1000 \\ yang@math.princeton.edu
}

These supporting institutions contribute to the cost of publication of this Journal, but they are not owners or publishers and have no responsibility for its contents or policies.

See inside back cover or msp.org/pjm for submission instructions.

The subscription price for 2014 is US $\$ 410 /$ year for the electronic version, and \$535/year for print and electronic.

Subscriptions, requests for back issues and changes of subscribers address should be sent to Pacific Journal of Mathematics, P.O. Box 4163, Berkeley, CA 94704-0163, U.S.A. The Pacific Journal of Mathematics is indexed by Mathematical Reviews, Zentralblatt MATH, PASCAL CNRS Index, Referativnyi Zhurnal, Current Mathematical Publications and Web of Knowledge (Science Citation Index).

The Pacific Journal of Mathematics (ISSN 0030-8730) at the University of California, c/o Department of Mathematics, 798 Evans Hall \#3840, Berkeley, CA 94720-3840, is published twelve times a year. Periodical rate postage paid at Berkeley, CA 94704, and additional mailing offices. POSTMASTER: send address changes to Pacific Journal of Mathematics, P.O. Box 4163, Berkeley, CA 94704-0163.

PJM peer review and production are managed by EditFLOW ${ }^{\circledR}$ from Mathematical Sciences Publishers.

\section{PUBLISHED BY}

\section{mathematical sciences publishers \\ nonprofit scientific publishing}

http://msp.org/

(C) 2014 Mathematical Sciences Publishers 


\section{PACIFIC JOURNAL OF MATHEMATICS}

Volume $271 \quad$ No. $1 \quad$ September 2014

Proper holomorphic maps between bounded symmetric domains revisited 1

GAUTAM BHARALI and JAIKRISHNAN JANARDHANAN

An explicit Majorana representation of the group $3^{2}: 2$ of $3 C$-pure type 25

HSIAN-YANG CHEN and CHING HUNG LAM

Sofic groups: graph products and graphs of groups

LAUra Ciobanu, DereK F. Holt and SARAh ReES

Perturbations of a critical fractional equation

Eduardo Colorado, Arturo de Pablo and Urko SÁnchez

A density theorem in parametrized differential Galois theory

THOMAS DREYFUS

On the classification of complete area-stationary and stable surfaces in $\quad 143$ the subriemannian Sol manifold

MATteo Galli

Periodic orbits of Hamiltonian systems linear and hyperbolic at infinity

BAŞAK Z. GÜREL

Nonsplittability of the rational homology cobordism group of

3-manifolds

SE-Goo KIM and CHARLES LIVINGSTON

Biharmonic surfaces of constant mean curvature

ERIC LOUBEAU and CEZAR ONICIUC

Foliations of a smooth metric measure space by hypersurfaces with constant $f$-mean curvature

JUNCHEOL PYO

On the existence of large degree Galois representations for fields of small 243 discriminant

JEREMY ROUSE and FRANK THORNE 